\title{
Ionic Basis for Plateau Potentials in Deep Dorsal Horn Neurons of the Rat Spinal Cord
}

\author{
Valérie Morisset and Frédéric Nagy \\ Institut National de la Santé et de la Recherche Médicale E.9914, Physiopathologie des Réseaux Neuronaux Médullaires, \\ Institut François Magendie, 33077 Bordeaux Cedex, France
}

Approximately $28 \%$ of dorsal horn neurons (DHNs) in lamina V of the rat spinal cord generate voltage-dependent plateau potentials underlying accelerating discharges and prolonged afterdischarges in response to steady current pulses or stimulation of nociceptive primary afferent fibers. Using intracellular recordings in a transverse slice preparation of the cervical spinal cord, we have analyzed the ionic mechanisms involved in the generation and maintenance of plateau potentials in lamina $\mathrm{V}$ DHNs. Both the accelerating discharges and afterdischarges were reversibly blocked by $\mathrm{Mn}^{2+}$ and enhanced when $\mathrm{Ca}^{2+}$ was substituted with $\mathrm{Ba}^{2+}$. The underlying tetrodotoxinresistant regenerative depolarization was sensitive to dihydropyridines, being blocked by nifedipine and enhanced by Bay $\mathrm{K}$ 8644. Substitution of extracellular $\mathrm{Na}^{+}$with $\mathrm{N}$-methyl-Dglucamine or choline strongly decreased the duration of the plateau potential. Loading the neurons with the calcium chelator BAPTA did not change the initial response but clearly decreased the maximum firing frequency and the duration of the afterdischarge. A similar effect was obtained with flufenamate, a specific blocker of the calcium-activated nonspecific cation current $\left(I_{\text {CAN }}\right)$. We conclude that the plateau potential of deep DHNs is supported by both $\mathrm{Ca}^{2+}$ influx through intermediatethreshold voltage-gated calcium channels of the L-type and by subsequent activation of a CAN current. $\mathrm{Ca}^{2+}$ influx during the plateau is potentially of importance for pain integration and the associated sensitization in spinal cord.

Key words: dorsal horn neurons; plateau potentials; bistability; afterdischarge; nociceptive integration; dihydropyridinesensitive intermediate voltage-activated $\mathrm{Ca}^{2+}$ current; CAN current; slice-intracellular technique
One correlate of central sensitization to pain is an increased background activity of spinal nociceptive neurons and the production of long-lasting afterdischarges. In vivo, afterdischarges are generated by deep dorsal horn neurons (DHNs) in response to nociceptive primary afferent inputs (Woolf and King, 1987; De Koninck and Henry, 1991). Their expression in response to peripheral stimulation is exaggerated in experimental models of persistent pain (Palecek et al., 1992; Laird and Bennett, 1993; Sotgiu et al., 1995; Grubb et al., 1996) and has been related to intense and prolonged behavioral responses to noxious stimuli (Laird and Bennett, 1993; Asada et al., 1996). These prolonged afterdischarges, apparently determinants for the perception of pain, are mediated in part by long-lasting excitatory synaptic potentials elicited in DHNs via the activation of neurokinin or amino acid receptors (Urban and Randic, 1984; Yoshimura and Jessell, 1990; De Koninck and Henry, 1991; Gerber et al., 1991; Nagy et al., 1993; Yoshimura et al., 1993).

In addition to synaptic components, however, intrinsic regenerative membrane properties of DHNs contribute significantly to the long-lasting nociceptive responses (Morisset and Nagy, 1996, 1998; Russo and Hounsgaard, 1996). We reported previously that $\sim 28 \%$ of deep dorsal horn neurons in the rat spinal cord exhib-

\footnotetext{
Received Dec. 7, 1998; revised June 10, 1999; accepted June 15, 1999.

This work was supported by grants from the DRET (95-148), the Conseil Régional d'Aquitaine (950301216), and the Institut UPSA de la Douleur. We are very grateful to Dr. D. Voisin and Dr. S. Oliet for careful reading and critical comments on this manuscript and to Dr. G. Le Masson for helpful discussions.

Correspondence should be addressed to Dr. Frédéric Nagy, Institut National de la Santé et de la Recherche Médicale E.9914, Physiopathologie des Réseaux Neuronaux Médullaires, Institut François Magendie, 1 rue Camille Saint-Saëns, 33077 Bordeaux Cedex, France.

Copyright (C) 1999 Society for Neuroscience $\quad 0270-6474 / 99 / 197309-08 \$ 05.00 / 0$
}

ited, in vitro, voltage-dependent plateau potentials positively modulated by the activation of metabotropic glutamate receptors (Morisset and Nagy, 1998). Expression of these regenerative depolarizations can profoundly alter the output properties of deep DHNs in response to sensory inputs. Nociceptive primary afferent stimulation elicited intense and prolonged responses in plateau-generating DHNs, whereas brief bursts of spikes were evoked in the absence of regenerative potential. Because plateau potentials had slow activation kinetics and were voltagedependent, plateau-generating neurons presented nonlinear input-output relationships in both the amplitude and time domains. Together, these results suggested that the ability of deep DHNs to generate plateau potentials might be crucial for the perception of pain in vivo. They also suggested that limiting the expression of regenerative membrane properties of DHNs is potentially of clinical interest as an alternative way of controlling pain-related central hyperexcitability. A prerequisite, however, is a reasonable knowledge of the ionic basis for these properties. Plateau potentials of deep DHNs were shown to depend on calcium (Morisset and Nagy, 1996), but the underlying conductances of the plateau were not precisely known in the rat.

Using a slice preparation from the cervical region of the rat spinal cord, we have analyzed in the present paper the membrane conductances involved in the generation and the maintenance of plateau potentials in lamina V DHNs. We show that the plateau potential is carried by both $\mathrm{Ca}^{2+}$ influx through voltage-gated calcium channels (VGCC) of the L-type and subsequent activation of a calcium-activated nonspecific cation current.

\section{MATERIALS AND METHODS}

The methods were described previously (Morisset and Nagy, 1996, 1998). Wistar rats of both sexes, aged from 17 to $26 \mathrm{~d}$, were anesthetized with 


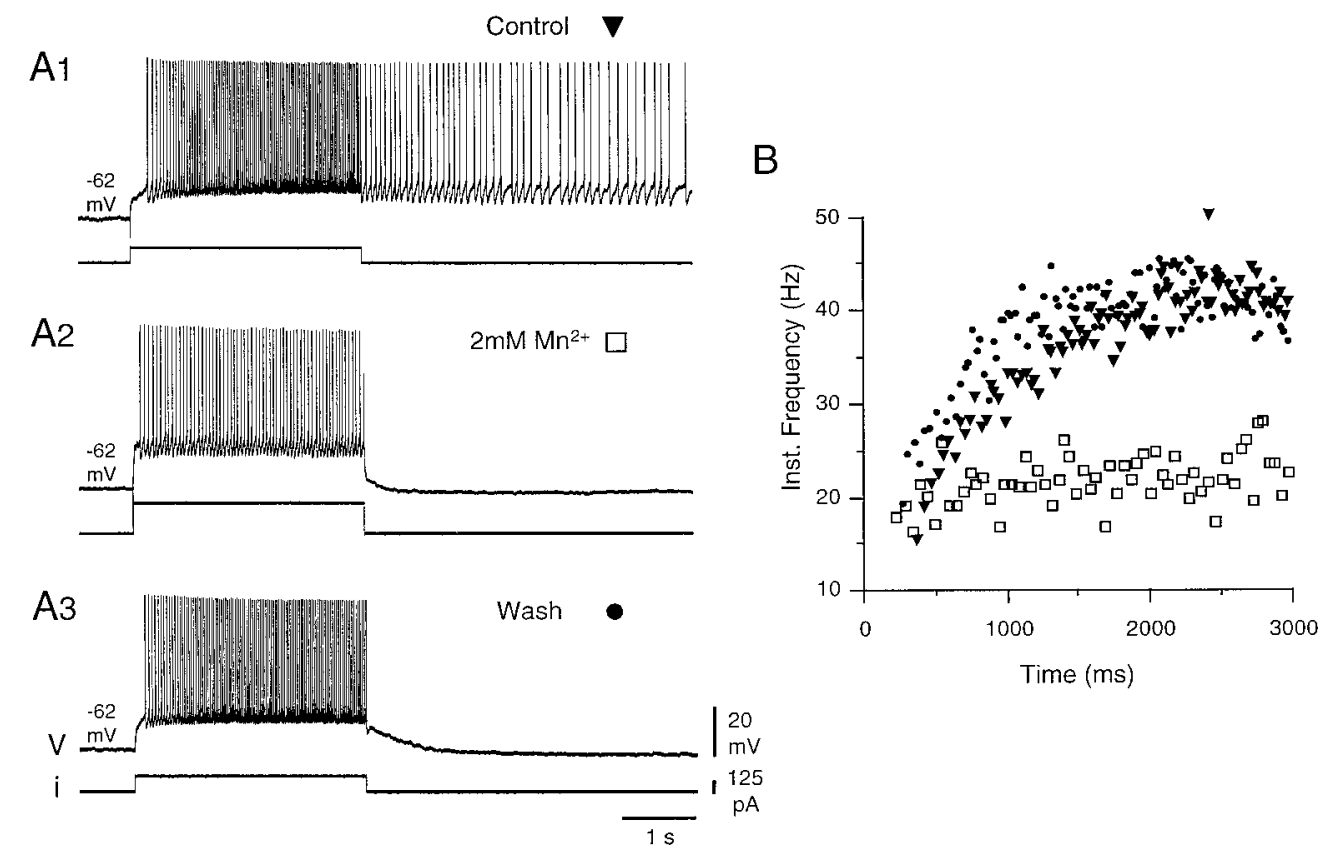

ether and decapitated. The excised cervical spinal cord was sliced transversally (400 $\mu \mathrm{m}$ sections) in the region C6-C8 using a vibratome (Campden Instruments Ltd, Leics, UK). The slices were transferred into the recording chamber on a layer of optical paper (interface-type chamber) on which they were perfused from below at a rate of $0.5 \mathrm{ml} / \mathrm{mn}$ with a Krebs' solution containing (in $\mathrm{mM}$ ): $124.0 \mathrm{NaCl}, 2.4 \mathrm{KCl}, 2.4 \mathrm{CaCl}_{2}, 1.3$ $\mathrm{Mg} \mathrm{SO}_{4}, 1.2 \mathrm{KH}_{2} \mathrm{PO}_{4}, 26.0 \mathrm{NaHCO}_{3}, 1.25 \mathrm{HEPES}$, and 10.0 glucose. The solution, maintained at a temperature of $30^{\circ} \mathrm{C}$, was oxygenated with $95 \% \mathrm{O}_{2}-5 \% \mathrm{CO}_{2}, \mathrm{pH}$ 7.4. Recording began after $2 \mathrm{hr}$ of equilibration.

Either a sharp electrode filled with $1 \%$ biocytin (Sigma, St. Quentin Fallavier, France) in $1 \mathrm{M}$ K-acetate (tip resistance of 160-200 M $\Omega$ ) or a patch electrode (tip resistance of 9-12 $\mathrm{M} \Omega$ ) was placed under visual control into the deep dorsal horn (lamina $\mathrm{V}$ of Rexed). The internal solution of patch pipettes had the following composition (in $\mathrm{mM}$ ): 120.0 K-gluconate, $20.0 \mathrm{KCl}, 0.1 \mathrm{CaCl}_{2}, 1.3 \mathrm{Mg} \mathrm{Cl}_{2}, 1.0$ EGTA, 10.0 HEPES, 0.1 GTP, 0.2 cAMP, 0.1 leupeptin, 3.0 Na - ATP, and 77.0 D-Mannitol, pH 7.3 (308 mOsm; 8 mOsm hyperosmotic to extracellular Krebs' solution). Signals were recorded with an Axoclamp 2B amplifier (Axon Instruments, Foster City, CA) and displayed on an oscilloscope (DSO 630; Gould, Ilford, Essex, UK) and a chart recorder (TA11; Gould). Acquisition and analysis were conducted with a Digidata 1200 system and the pClamp 6 software (Axon Instruments) connected to a 486 IBMcompatible computer. Current injection was controlled by the Digidata 1200 system or by a stimulator (Master 8; AMPI, Jerusalem, Israel). Current was injected into the neurons through the same electrode via a bridge circuit in the amplifier. Bridge balance was monitored throughout experiments. Subsequent data analysis was performed with the pClamp 6 software (Axon Instruments), Excel 5.0 (Microsoft, Seattle, WA) and SigmaPlot 4.16 (SPSS Inc., Chicago, IL). During electrophysiological recordings with sharp electrodes, neurons were filled with biocytin for subsequent morphological characterization. The morphological characteristics and the types of sensory input integrated by the plateaugenerating deep dorsal horn cells have been presented previously (Morisset and Nagy, 1998). Mean resting membrane potential and neuronal input resistance were calculated for a subset of the recorded neurons (resting membrane potential, $-57.7 \pm 1.2 \mathrm{mV}$; input resistance, $98.7 \pm$ $6.5 \mathrm{M} \Omega$; mean $\pm \mathrm{SD} ; n=30$ ).

When needed, the following drugs were added to the normal Krebs' solution and continuously superfused on the preparation: $1 S, 3 R-1$ amino-1,3-cyclopentanedicarboxylic acid (1S,3R-ACPD), ( \pm )-2-amino5-phosphonopentanoic acid (AP-5), apamin, bicuculline, and 6-cyano-7nitroquinoxaline-2,3-dione (CNQX) were from Research Biochemicals (Natick, MA); BAPTA-AM was from Calbiochem (La Jolla, CA); cAMP, $\mathrm{Na}_{2}$-ATP, Bay-K 8644, choline chloride, EGTA, flufenamic acid (FFA), GTP, HEPES, leupeptin, nifedipine, $N$-methyl-D-glucamine (NMDG), strychnine, and tetrodotoxin (TTX) were from Sigma. Nifed- ipine and FFA were freshly dissolved in dimethylsulfoxide (DMSO) for each experiment. Care was taken to protect nifedipine from light. DMSO had no effects per se at the concentration used $(0.1$ and $0.2 \%$, respectively). Low-sodium saline consisted of normal perfusion medium in which $124.0 \mathrm{~mm} \mathrm{NaCl}$ was replaced with $124.0 \mathrm{~mm}$ NMDG or $124.0 \mathrm{~mm}$ choline chloride. $\mathrm{Mn}^{2+}$ was added to a modified perfusion medium containing (in mM): $124.0 \mathrm{NaCl}, 3.6 \mathrm{KCl}, 2.4 \mathrm{CaCl}_{2}, 1.3 \mathrm{Mg} \mathrm{Cl}_{2}, 26.0$ $\mathrm{NaHCO}_{3}, 1.25$ HEPES, and 10.0 glucose. $\mathrm{Ba}^{2+}$ saline consisted of the same modified medium in which $2.4 \mathrm{CaCl}_{2}$ was substituted with equimolar $\mathrm{BaCl}_{2}$. When needed in the presence of a drug, bias current was injected to keep same holding potential or to reach same initial firing frequency as in control. Data presented in all the figures were obtained in the presence of a mixture of $50 \mu \mathrm{M}$ AP-5, $20 \mu \mathrm{M}$ CNQX, $20 \mu \mathrm{M}$ bicuculline, and $50 \mu \mathrm{M}$ strychnine to block NMDA receptors and most of the fast excitatory and inhibitory synaptic transmission. In Figures 2 and 4-6, plateau potentials were induced in the presence of the metabotropic glutamate receptor agonist 1S,3R-ACPD (Morisset and Nagy, 1996, 1998). Recordings were obtained with patch pipettes (whole-cell configuration) in Figure 6 and with sharp electrodes in all other figures.

\section{RESULTS \\ Nifedipine-sensitive $\mathrm{Ca}^{2+}$ component of the plateau potential}

In plateau-generating DHNs, both the acceleration of firing during the injection of a pulse of depolarizing current (Fig. $1 A 1, B$, filled triangles) and the afterdischarge were suppressed in the presence of $2 \mathrm{mM} \mathrm{Mn}^{2+}$, which is known to block voltageactivated calcium currents (Fig. 1A2,B, open squares) $(n=3)$. Note that the intensity of the current pulse was adjusted to elicit the same initial firing frequency as in the control (mean firing frequency of 18.6 and $19.3 \mathrm{~Hz}$, respectively, during the first 100 msec of the discharge). Nevertheless, the subsequent evolution of the discharges was radically different (mean firing frequency during the last $100 \mathrm{msec}$ of the discharge of $40.9 \mathrm{~Hz}$ in control and 20.6 $\mathrm{Hz}$ in the presence of $\mathrm{Mn}^{2+}$ ). The block by $\mathrm{Mn}^{2+}$ was reversible (Fig. 1A3, 1B, filled circles). Conversely, plateau potentials were enhanced when $\mathrm{Ca}^{2+}$ was substituted with $\mathrm{Ba}^{2+}$ in the bathing medium (four of four DHNs). Barium is a better charge carrier through VGCCs, does not activate $\mathrm{Ca}^{2+}$-dependent $\mathrm{K}^{+}$ conductances, and subsequently blocks most of the potassium channels from the inner face of the membrane. The effects of the 

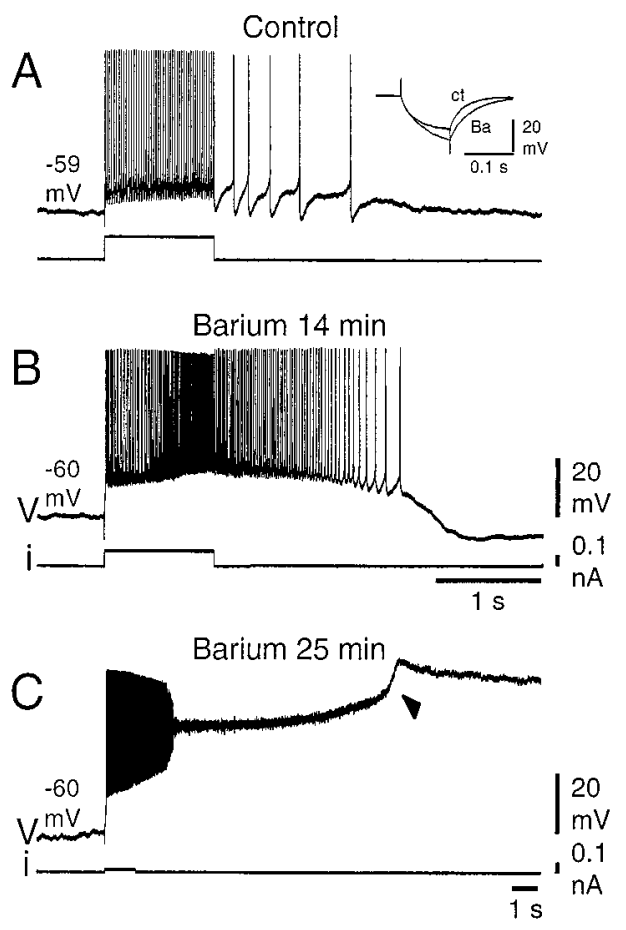

Figure 2. Plateau-potential amplification in the presence of barium. $A$, In control conditions, a DHN responded to a $1 \mathrm{sec}$ depolarizing current pulse with a discharge of moderate acceleration, followed by a weak afterdischarge. $B$, After $14 \mathrm{~min}$ in the barium solution $\left(\mathrm{Ca}^{2+}\right.$ substituted with equimolar $\mathrm{Ba}^{2+}$ ), the latter were substantially increased. Barium also caused an increase in the neuron input resistance $(A$, inset $)$. $C$, After a more prolonged exposure to the barium solution $(25 \mathrm{~min})$, the amplitude of the plateau potential was enhanced, leading to inactivation of action potentials, and a long-lasting afterdepolarization was produced. Note after $4.5 \mathrm{sec}$ a further depolarizing step in the plateau potential (arrowhead). Recordings were made in the presence of the mGluR agonist $1 S, 3 R$-ACPD $(25 \mu \mathrm{M})$.

substitution are illustrated in Figure 2 for a neuron that expressed moderate acceleration of firing and reduced afterdischarge in response to a pulse of depolarizing current in control conditions (Fig. 2A). After $14 \mathrm{~min}$ in the presence of barium (Fig. 2B), the firing frequency during and after the stimulation was much higher $(118.6 \pm 9.7 \mathrm{~Hz} ; n=3$ stimulations during the last $100 \mathrm{msec}$ of the stimulation in $\mathrm{Ba}^{2+} ; 46.7 \pm 1.4 \mathrm{~Hz}, n=3$ in control), and the afterdischarge was longer. A longer exposure to the $\mathrm{Ba}^{2+}$ solution further increased the amplitude and duration of the plateau potentials, leading to spike inactivation (Fig. 2C). These enhanced plateau potentials obtained in the presence of $\mathrm{Ba}^{2+}$ could still be repolarized by the injection of hyperpolarizing current pulses (data not shown).

As shown previously (Morisset and Nagy, 1996), the $\mathrm{Ca}^{2+}$ dependent bistability was also maintained under $1 \mu \mathrm{M}$ tetrodotoxin $(n=24)$, further indicating that the ability to produce plateau potentials is an endogenous membrane property of the deep DHNs. Under these conditions, a slow-rising plateau potential still developed during injection of square pulse of depolarizing current and was followed by a long-lasting afterdepolarization (Figs. 3A1, control) that could be interrupted by a brief pulse of hyperpolarizing current (data not shown).

TTX-resistant plateau potentials were also highly sensitive to dihydropyridines, which are known to modulate negatively (nifedipine) and positively ( $S$-Bay K 8644) L-type calcium currents (Fox et al., 1987b). They were almost completely blocked by bath-applied nifedipine ( $1 \mu \mathrm{M}, n=2$ of $2 ; 10 \mu \mathrm{M}, n=9$ of 9 ) (Fig. $3 A 1)$. Conversely, both the amplitude of the plateau during the stimulation and the duration of the afterdepolarization were enhanced by $2 \mu \mathrm{M} S$-Bay K 8644 (Fig. $3 B 1)$ ( $n=3$ of 3). The $I-V$ plots obtained at the end of $3 \mathrm{sec}$ depolarizing current pulses of increasing intensities in the presence of nifedipine $(10 \mu \mathrm{M})$ (Fig. $3 A 2$ ) or $S$-Bay K 8644 (Fig. 3B2) begin to differentiate from the control $I-V$ plots at approximately $-55 \mathrm{mV}$. The threshold for the dihydropyridine-sensitive current was therefore in agreement with the plateau-potential threshold measured in normal saline $(-53.9 \pm 1.3 \mathrm{mV} ; n=6)$. Both the regenerative depolarization during the stimulation and the afterdepolarization were correlated with a strong decrease in membrane resistance (Fig. 3C), whereas the slow repolarizing phase after the plateau corresponded to a progressive recovery of the control value. The preceding results demonstrate that bistability in DHNs is supported by a nifedipine-sensitive $\mathrm{Ca}^{2+}$-dependent plateau potential. Accordingly, in the absence of TTX, both the acceleration of firing during injection of a depolarizing current pulse and the afterdischarge were suppressed in the presence of nifedipine $(n=$ 3 of 3; data not shown). Together, our results indicate that L-type calcium channels are involved in the endogenous plateau properties of deep DHNs underlying accelerating discharges and prolonged afterdischarges.

\section{$\mathrm{Ca}^{2+}$-dependent depolarizing component of the plateau potential}

Because $\mathrm{Ca}^{2+}$ entry through VGCCs could subsequently activate calcium-dependent depolarizing conductances, such as a calciumactivated nonspecific cationic conductance $\left(I_{\mathrm{CAN}}\right)$ (Swandulla and Lux, 1985; Partridge et al., 1994), we addressed the question of whether the calcium was in itself a charge carrier responsible for the plateau potential or whether the generation and maintenance of regenerative depolarizations were caused, in part or totally, by $\mathrm{Ca}^{2+}$-dependent conductances.

In a first series of experiments, we have examined the effect of loading plateau-generating neurons with a calcium chelator that prevents the activation of $\mathrm{Ca}^{2+}$-dependent conductances. Figure 4 shows that applying in the bath $50 \mu \mathrm{M}$ of the membranepermeable calcium chelator BAPTA-AM clearly decreased the expression of plateau potentials. A DHN that produced an accelerating discharge and an afterdischarge in response to a $3 \mathrm{sec}$ depolarizing current pulse in control conditions (Fig. 4A1) became unable to generate the afterdischarge, regardless of the membrane potential in the presence of BAPTA-AM (Fig. 4A2). Interestingly, in the presence of the calcium chelator, the discharge was still accelerating during the stimulation. However, analysis of the instantaneous firing frequency (Fig. 4A3, open circles) shows that, although initially similar to the control (Fig. $4 A 3$, filled circles), the firing acceleration stopped after $1.5 \mathrm{sec}$ (Fig. 4A3, dashed line), and the mean firing frequency during the last $100 \mathrm{msec}$ of the stimulation was lower $(28.0 \mathrm{~Hz})$ than in control $(44.8 \mathrm{~Hz})$. Therefore, in the presence of BAPTA-AM, a regenerative depolarization was still observed, but a delayed depolarizing component of the response disappeared, preventing the afterdischarge. The fact that different components participate in the initial acceleration of firing and in the afterdischarge appears more clearly in Figure 4, $B$ and $C$, in which another DHN was stimulated with shorter current pulses. In the control conditions (Fig. 4B1), the neuron produced an afterdischarge over $>30$ sec. After the end of the stimulus, the firing frequency raised gradually to peak after $10 \mathrm{sec}$ at $14.4 \mathrm{~Hz}$ (mean frequency over 0.5 
Figure 3. TTX-resistant plateau potentials were sensitive to dihydropyridines. $A 1$, The plateau potential was blocked in the presence of $1 \mu \mathrm{M}$ nifedipine (traces in control and nifedipine superimposed). $A 2$, The $I-V$ plot obtained from another neuron (potential measured at the end of $800 \mathrm{msec}$ hyperpolarizing and depolarizing current pulses injected from a holding potential of $-56 \mathrm{mV}$ ) shows that the nifedipine-sensitive component of the plateau potential activated at approximately $-55 \mathrm{mV}$. B1, The plateau potential was increased in both amplitude and duration in the presence of 2 $\mu \mathrm{M}$ Bay K 8644. B2, The $I-V$ plot obtained from the same neuron again shows that the threshold of the Bay K 8644-sensitive component was approximately $-55 \mathrm{mV}$. C1, The neuron input resistance decreased during the development of the plateau potential and progressively recovered during the repolarization. C2, Voltage deflections in response to $25 \mathrm{msec}$ hyperpolarizing pulses shown at larger time and amplitude scales before $(1)$ and during $(2,3)$ the depolarizing phase of the plateau (same records as in C1). All the recordings were obtained in the presence of 1 $\mu \mathrm{M}$ TTX. $B, C$, Same neuron. $A 1, A 2, B$, Different neurons.

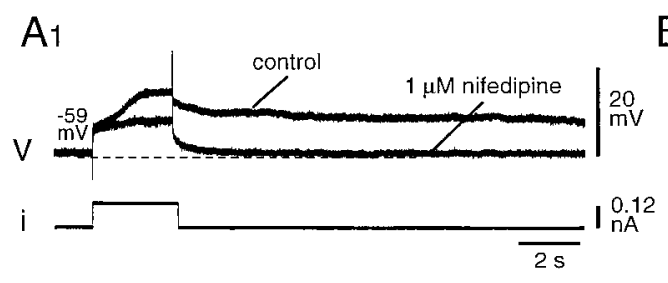

B1

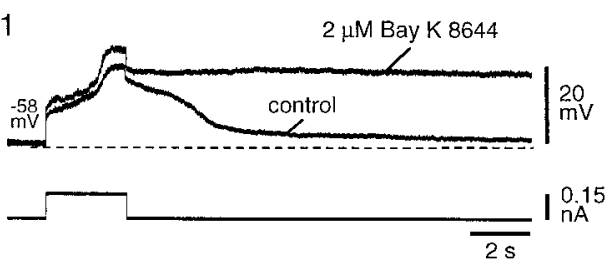

A2
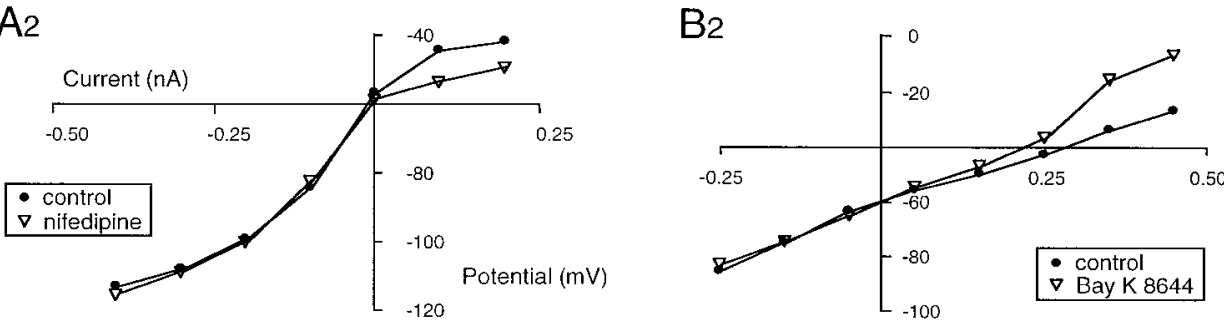

$2 \mu \mathrm{M}$ Bay K 8644

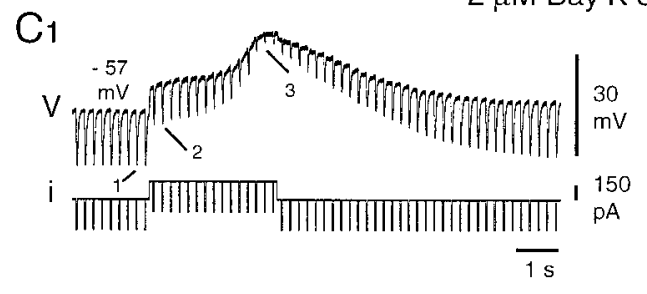

$\mathrm{C} 2$

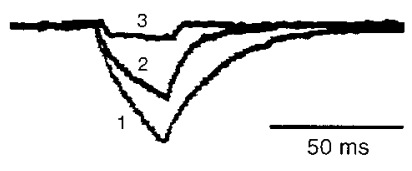

sec) yielding to a mean frequency of $10.8 \mathrm{~Hz}$ during the first 20 sec of the afterdischarge. After $40 \mathrm{~min}$ in the presence of $50 \mu \mathrm{M}$ BAPTA-AM (Fig. 4B2), the afterdischarge produced in response to the same stimulation lasted for $14.9 \mathrm{sec}$ with a much lower firing frequency $(2.5 \mathrm{~Hz})$. After $1 \mathrm{hr} 40 \mathrm{~min}$ in the presence of the chelator, no afterdischarge was generated, regardless of the membrane potential (Fig. 4B3). Interestingly, in the three situations, the same firing pattern was produced during the stimulation (mildly accelerating discharge of $\sim 20 \mathrm{~Hz}$ ) (Fig. $4 C$ ). Moreover, in the same conditions as in Figure $4 B 3$ ( $1 \mathrm{hr} 40 \mathrm{~min}$ in BAPTAAM), a shorter stimulation $(0.35 \mathrm{sec})$ (Fig. $4 B 4)$ was able to elicit an afterdischarge. Most probably, during the brief stimulation, the cumulative spike-associated afterhyperpolarizing potential did not develop enough to counteract the calcium currentmediated depolarization. The afterdischarge, however, was shorter than $8 \mathrm{sec}$ with a mean firing frequency of $3.4 \mathrm{~Hz}$. Similar results were obtained in three of three neurons. Together, they indicate that the plateau potential in deep DHNs is supported by both an L-type $\mathrm{Ca}^{2+}$ current and a $\mathrm{Ca}^{2+}$-activated depolarizing current and that the calcium current by itself can only sustain a mild and relatively short afterdischarge, if any.

In a second series of experiments, we investigated further the type of calcium-dependent current involved by testing the possible implication of a calcium-activated nonspecific cationic current. For that purpose, plateau potentials were tested in mediums in which $\mathrm{Na}^{+}$was substituted with NMDG or choline. The former substance is known to have very low permeability through the CAN channels (Bal and McCormick, 1993; Wilson et al., 1996). The permeability of choline varies depending on the neuronal type (Partridge et al., 1994; Rekling and Feldman, 1997). These substitutions were performed in the presence of $1 \mu \mathrm{M}$ TTX. Figure $5 A 2$ shows that, when $\mathrm{Na}^{+}$was substituted with NMDG, the peak depolarization during the current injection, as well as the afterdepolarization after the stimulation, were clearly re- duced in amplitude compared with the control (Fig. 5A1). Figure $5 B$ illustrates a similar effect for another type of plateaugenerating DHN, producing only very short afterdepolarization in control. Again, the peak depolarization during the stimulation was reduced in the NMDG medium (Fig. 5B2), the reduction being reversible (Fig. 5B3). The effects of NMDG substitution were obtained in three of three neurons. Similar reduction of the plateau potential was also obtained in three of three other DHNs when $\mathrm{Na}^{+}$was substituted with choline. In the example of Figure $5 C$, in control conditions (Fig. 5C1), the duration of the plateau potential was variable but always longer than $10 \mathrm{sec}(n=6$ stimulations), whereas in the choline medium, it fell to $3.5 \pm 0.2$ sec $(n=6)$ when elicited from the same holding potential (Fig. 5C2). Again, the effect was reversible (Fig. 5C3). Note in Figure $5 C 2$ the difference between a subthreshold (left) and a suprathreshold (right) stimulation. The residual regenerative depolarization may be attributable to either partial permeability of both NMDG and choline through CAN channels or calcium current through the L-type VGCC.

Participation of a CAN current in the plateau potential was further investigated by using FFA, a specific antagonist of $I_{\text {CAN }}$ (Shaw et al., 1995). Figure 6 shows that the late phase of the plateau potential was strongly reduced in the presence of $0.5 \mathrm{~mm}$ FFA. In the example of Figure $6 A$, the neuron responded to a 1 sec depolarizing current pulse with an afterdischarge longer than $20 \mathrm{sec}$ (Fig. 6A1). In the presence of FFA, no afterdischarge was obtained (Fig. 6A2), regardless of the holding potential or the stimulus intensity. Again, however, there was no clear difference in the type of firing pattern during the stimulation compared with control. As indicated by the slope of the regression lines in Figure $6 A 3$, during the first second of firing, the acceleration was 8.0 $\mathrm{Hz} / \mathrm{sec}$ in control and $9.4 \mathrm{~Hz} / \mathrm{sec}$ in the presence of FFA (linear regression coefficients, 0.68 in both cases). The early and late phases of the plateau have, therefore, the same different sensitiv- 

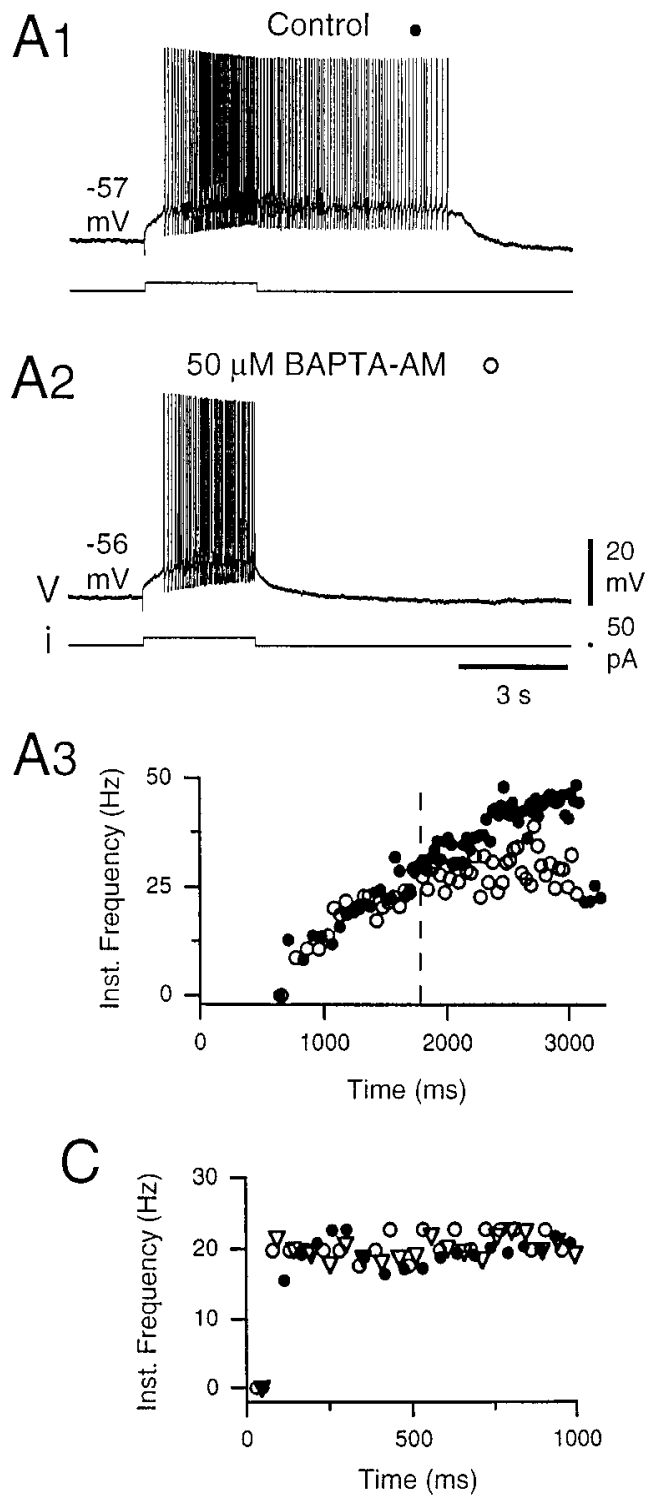
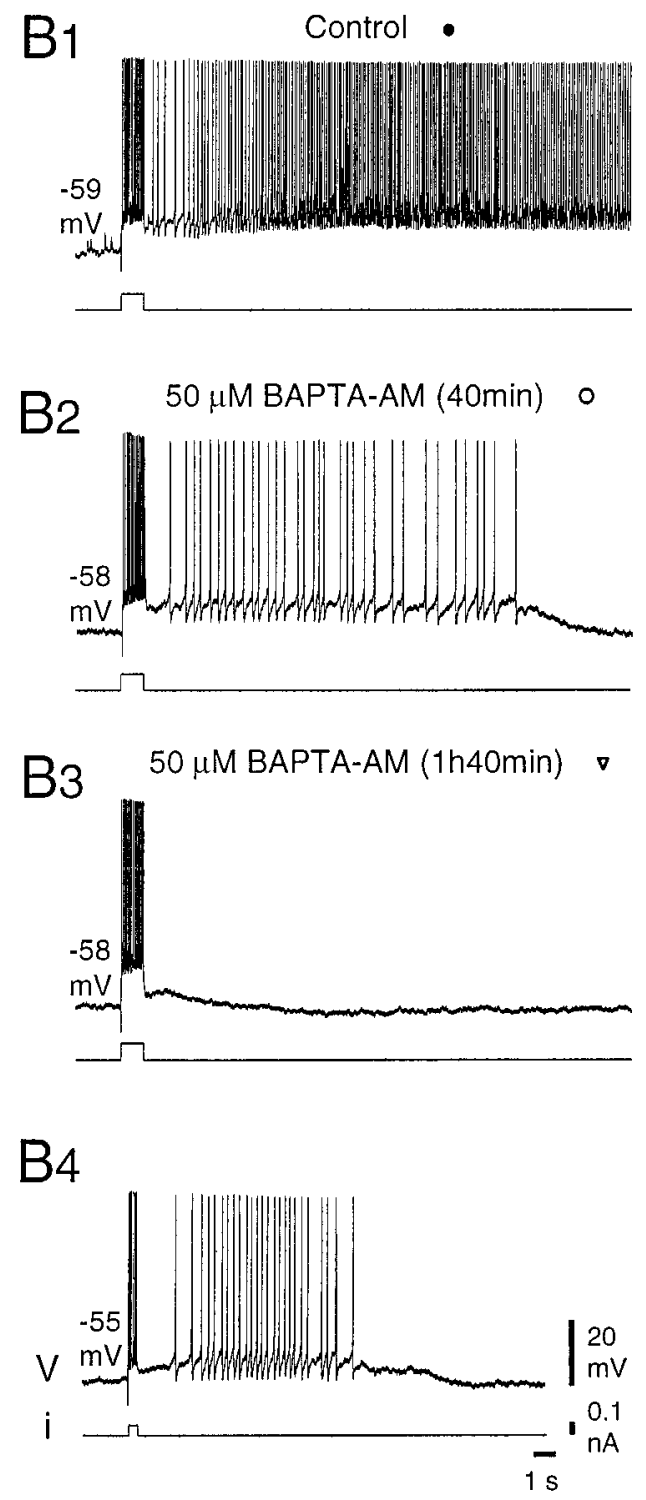

Figure 4. Chelating intracellular calcium decreased the late phase of plateau potentials. $A$, In control conditions, the neuron responded to a $3 \mathrm{sec}$ pulse of depolarizing current with an accelerating discharge, followed by an afterdischarge (A1, A3, filled circles). Application of the calcium chelator BAPTA-AM $(50 \mu \mathrm{M})$ did not change the initial response of the neuron ( $A 3$, open circles) but interrupted the acceleration of discharge after $1.5 \mathrm{sec}(A 3$, dashed line) and abolished the afterdischarge (A2). $A 3$, Instantaneous frequency plot calculated as in Figure 1 for the discharges recorded in $A 1$ and $A 2$. $B$, In control conditions, a longlasting and intense plateau potential was triggered by a short current pulse $(1 \mathrm{sec})$ in another DHN (B1). Note the slow increase in firing frequency during the afterdischarge. In the presence of BAPTA-AM, the duration and intensity of the afterdischarge gradually decreased (B2, $40 \mathrm{~min})$, until it disappeared (B3, $1 \mathrm{hr} 40 \mathrm{~min})$. Conversely, the firing frequency during the $1 \mathrm{sec}$ stimulation did not change $(C$, instantaneous frequency plot calculated for B1-B3). Under the same conditions, a weak afterdischarge could be triggered by a shorter pulse of current (B4). All the recordings were obtained in the presence of the mGluR agonist $1 S, 3 R$ $\operatorname{ACPD}(25 \mu \mathrm{M}) \cdot A, B$, Different neurons. ity to FFA as to BAPTA (Fig. $4 A, B$ ). In the case of the neuron in Figure $6 B$, the stimulus triggered a short plateau that lasted only for $5.5 \mathrm{sec}$ and led to an intense firing during the afterdischarge (Fig. 6B1, 6B3, peak at $47.6 \mathrm{~Hz}$, filled circles). In response to the same stimulation in the presence of $0.5 \mathrm{~mm}$ FFA (Fig. 6B2), the maximum firing frequency during the afterdischarge was much lower $(16.1 \mathrm{~Hz})$ (Fig. 6B3, open circles), although the mean firing frequency during the first second of the discharge was similar to the control (9.6 and $9.9 \mathrm{~Hz}$, respectively). Unexpectedly, the afterdischarge was prolonged in the presence of FFA. This was most probably caused by a weaker afterhyperpolarizing potential associated with the much lower depolarization during the plateau potential. Strong reduction of the plateau potential was obtained in four of four plateau-generating neurons.

In summary, our results demonstrate that the plateau potential in deep DHNs is supported by both an L-type $\mathrm{Ca}^{2+}$ current and a calcium-activated nonspecific cationic current. The former is the major depolarizing component during the initial phase of the plateau, and the latter is necessary for the expression of longlasting afterdischarges.

\section{DISCUSSION}

Expression of voltage-dependent plateau potentials by dorsal horn neurons in response to the stimulation of primary afferent fibers is of particular importance for the processing of nociceptive information in the spinal cord (Morisset and Nagy, 1996, 1998; Russo and Hounsgaard, 1996). In the present study, we analyzed the ionic basis for the intrinsic regenerative properties of the rat DHNs.

\section{Generation and maintenance of the plateau potential}

Plateau potentials, or more generally regenerative depolarizations outlasting the duration of a stimulus, have been described in a variety of neuronal types in both invertebrates (Golowasch and Marder, 1992; Kiehn and Harris-Warrick, 1992; Zhang and Harris-Warrick, 1995; Zhang et al., 1995; Angstadt and Choo, 1996; Wilson et al., 1996; Mills and Pitman, 1997) and vertebrates (Hounsgaard and Mintz, 1988; Kiehn, 1991; Fraser and MacVicar, 1996; Russo and Hounsgaard, 1996; Overton and Clark, 1997; Rekling and Feldman, 1997; Viana di Prisco et al., 1997; Hsiao et al., 1998; Sandler et al., 1998), and the mechanisms 


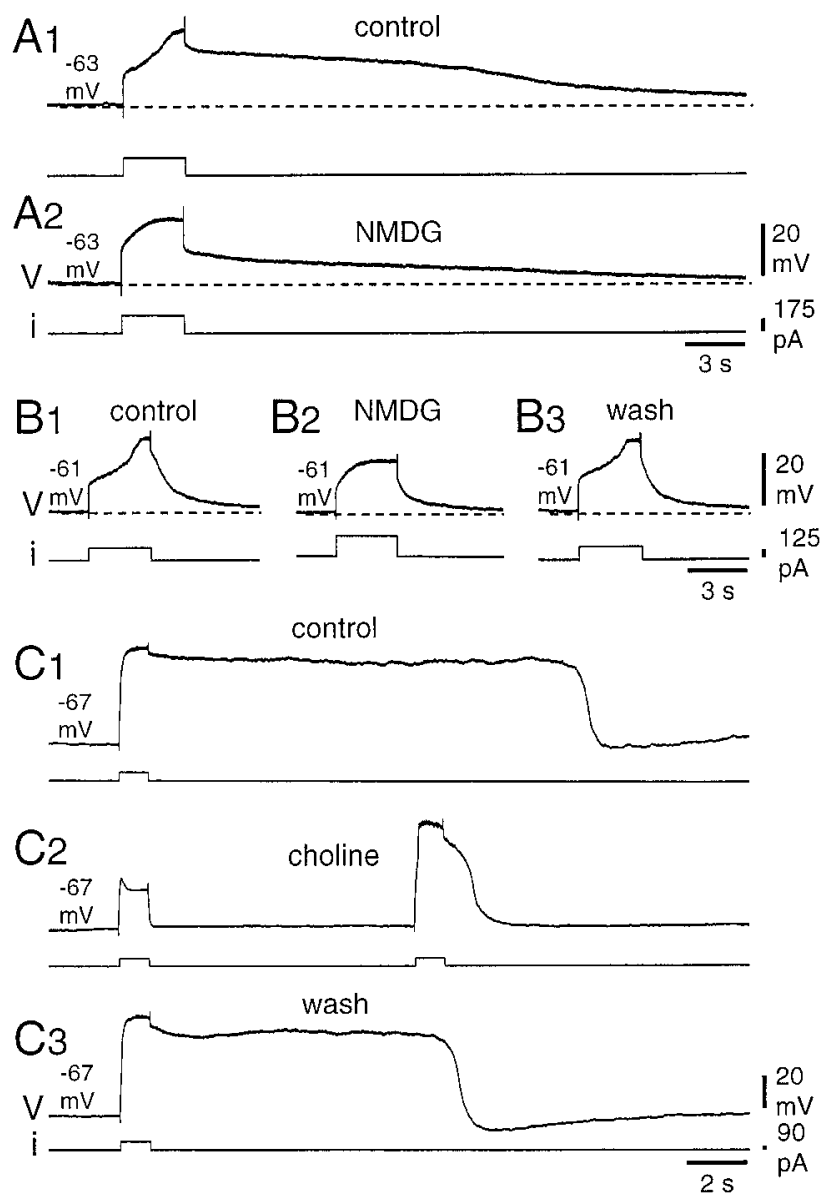

Figure 5. Sodium substitution decreased the amplitude and duration of TTX-resistant plateau potentials. All the recordings were obtained in the presence of $1 \mu \mathrm{M}$ TTX and $50 \mu \mathrm{M} 1 S, 3 R$-ACPD. $A$, When most of the $\mathrm{Na}^{+}$was replaced by NMDG in the perfusion medium, the amplitude of both the late depolarizing phase during the stimulation and the afterdepolarization were reduced $(A 2)$ compared with control conditions $(A 1)$. In neurons producing short plateaus $(B 1)$, the NMDG medium reduced the late phase of the regenerative depolarization $(B 2)$. The reduction was reversible $(B 3)$. $C$, Similar reversible effects were obtained when most of the $\mathrm{Na}^{+}$was replaced by choline in the bath. In the choline medium, note the difference between a subthreshold ( $C 2$, first stimulation) and a suprathreshold response (second stimulation).

are diverse. The present data show that, in DHNs of the rat, the plateau potential is calcium-dependent, being both resistant to TTX and blocked by $\mathrm{Mn}^{2+}$. In addition, maximum firing frequency and duration of the afterdischarge supported by the plateau are strongly reduced when intracellular $\mathrm{Ca}^{2+}$ concentration is maintained low by the calcium chelator BAPTA, indicating that the plateau potential is supported by both a $\mathrm{Ca}^{2+}$ conductance and a $\mathrm{Ca}^{2+}$-dependent conductance. The calcium chelator was applied in the bath under its membrane-permeable form BAPTA-AM. Therefore, it could have also perturbed a spontaneous release of transmitters in the slice preparation, whereby inducing indirect modifications of the DHNs regenerative properties. However, our experiments conducted in a mixture of synaptic blockers eliminated the possibility of indirect effects via ionotropic neurotransmission. The indirect decrease of plateau properties via modifications in the release of neuromodulators is also unlikely because the initial part of the plateaus was almost unchanged in the presence of the chelator. Moreover, ionic substitutions and application of blockers confirmed the implication of a $\mathrm{Ca}^{2+}$-dependent conductance in the plateau potential of the deep DHNs.

Application of these substances indicated that the calciumdependent conductance involved in the depolarizing phase of the plateau potential was a $\mathrm{Ca}^{2+}$-activated nonselective cation current $\left(I_{\mathrm{CAN}}\right)$ (Partridge et al., 1994), which was shown in a variety of neurons to sustain burst discharge, afterdischarge, and plateau potentials, or oscillations (Swandulla and Lux, 1985; Zhang et al., 1995; Fraser and MacVicar, 1996; Wilson et al., 1996; Klink and Alonso, 1997; Viana di Prisco et al., 1997; Beurrier et al., 1999). In the rat DHNs, the amplitude and/or the duration of the plateau potential were strongly reduced when most of extracellular $\mathrm{Na}^{+}$was replaced by impermeable molecules through the CAN channels (NMDG or choline), or during superfusion with the specific $I_{\text {CAN }}$ blocker FFA. FFA, one member of a class of nonsteroidal anti-inflammatory drugs, was shown to affect two calcium-activated conductances in neurons of the snail Helix aspersa (Shaw et al., 1995). It induced a transient increase in $I_{\mathrm{CAN}}$ and in a calcium-activated chloride current, consecutive to a rise in intracellular calcium concentration; subsequently, it blocked the two calcium-activated conductances. To our knowledge, no calcium-activated chloride current was described in the DHNs, and, in any case, blockade of such a hyperpolarizing conductance by FFA would have prolonged plateau potentials. On the other hand, a CAN current was reported in a proportion of rat DHNs (Murase et al., 1989), and the clear and permanent decrease of the late phase of the plateaus in the presence of FFA, together with the effects of $\mathrm{Ca}^{2+}$ chelation and $\mathrm{Na}^{+}$substitutions, indicates that $I_{\mathrm{CAN}}$ is actually involved in this late depolarizing phase of the plateau potentials. Dynamic interactions between $I_{\mathrm{CAN}}$ and intermediate-to-high threshold $\mathrm{Ca}^{2+}$ currents were reported in other plateau-producing neurons (Zhang et al., 1995; Fraser and MacVicar, 1996). In the latter cases, blocking the $I_{\text {CAN }}$ completely eliminated the plateau potentials. When $I_{\text {CAN }}$ was blocked in DHNs, however, the reduction only concerned the late phase of the plateau, leaving the early phase unchanged. Moreover, a weak afterdischarge can still show up in the presence of BAPTA. Therefore, $I_{\mathrm{CAN}}$ was not the only depolarizing component of the regenerative plateau potential.

Our data indicate that the plateau potential in DHNs of the rat spinal cord was also carried by calcium influx through VGCC, which activated a few millivolt positive to resting membrane potential. This intermediate threshold calcium current was mediated probably by L-type calcium channels, being sensitive to dihydropyridines. Plateau potentials supported by similar calcium currents were described in DHNs of the turtle spinal cord (Russo and Hounsgaard, 1996) and various motoneurons (Hounsgaard and Mintz, 1988; Hsiao et al., 1998). In the latter cases, as well as in the rat DHNs (present paper), the involved L-type calcium current activated at a more negative membrane potential than for typical L-type currents (Fox et al., 1987a; Tsien et al., 1988). Dihydropyridine sensitivity was reported for a number of low- or intermediate-voltage-activated calcium currents, showing little or no inactivation (Marchetti et al., 1995; Avery and Johnston, 1996; Kavalali and Plummer, 1996). In DHNs of the rat spinal cord, voltage-clamp studies described modulation by dihydropyridines of a sustained $\mathrm{Ca}^{2+}$ current for membrane potentials positive to $-50 \mathrm{mV}$ (Huang, 1989; Ryu and Randic, 1990). Interestingly, both this intermediate-threshold $\mathrm{Ca}^{2+}$ current and an $I_{\mathrm{CAN}}$ were reported to be activated or enhanced by substance $\mathrm{P}$ in a proportion of the rat DHNs (Murase et al., 1989; Ryu and Randic, 1990), 

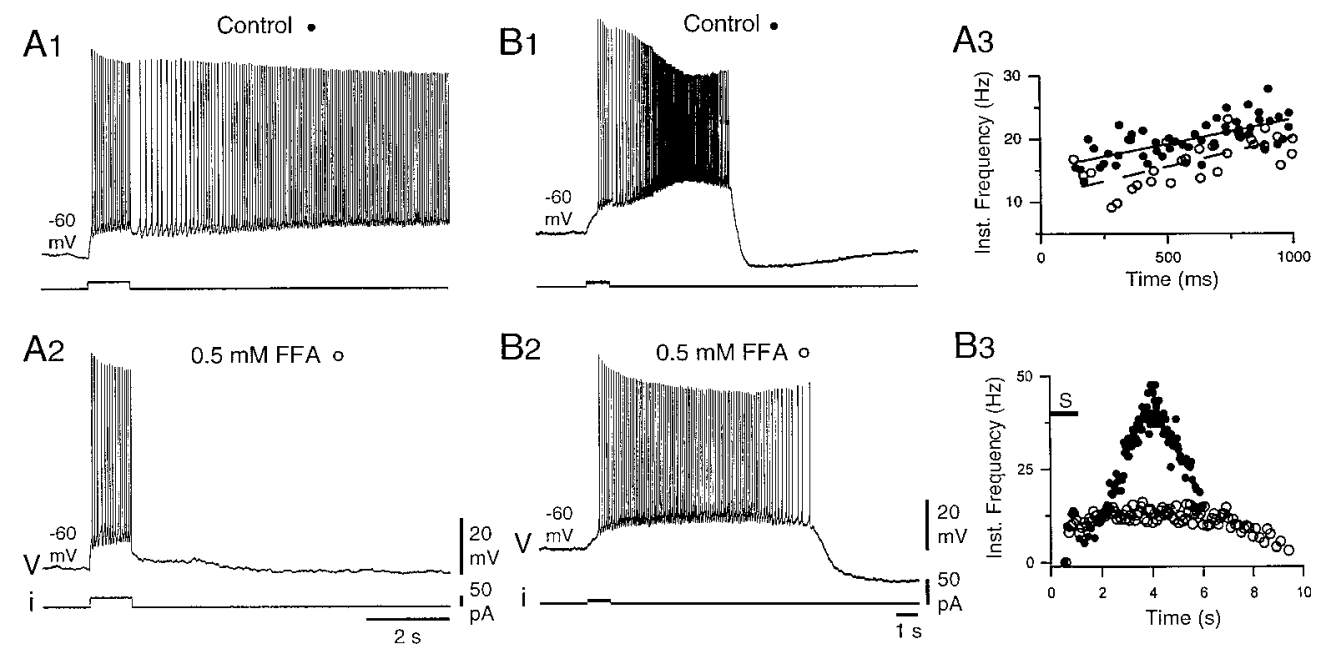

Figure 6. The $I_{\text {CAN }}$ blocker FFA blocked the late phase of plateau potentials. $A$, In control conditions, a full plateau potential supported a prolonged afterdischarge. Again, note the progressive firing acceleration after termination of the $1 \mathrm{sec}$ stimulation. The afterdischarge was suppressed in the presence of $0.5 \mathrm{~mm}$ FFA $(A 2)$, although the firing pattern during the stimulation was not affected ( $A 3)$. $A 3$, The instantaneous frequency plot was calculated as in Figure 1 for two discharges in control conditions ( filled circles) and for two discharges in the presence of FFA (open circles). Lines are linear regressions through the data points (solid line, control; dashed line, FFA) (correlation coefficients, 0.68 in both cases; see Results). $B$, In another DHN for which the plateau quickly repolarized (B1), application of FFA strongly reduced the maximum firing frequency and prolonged the duration of the afterdischarge (B2, B3, open circles) (see Results). B3, The instantaneous frequency plots were calculated for the discharges in $B 1$ and $B 2(S, 1$ sec depolarizing pulse). All recordings were obtained in the presence of $25 \mu \mathrm{M} 1 S, 3 R$-ACPD.

just like the plateau potential (Russo et al., 1997). A possibility remains that $I_{\mathrm{CAN}}$ is activated by other sources of calcium. Highvoltage-activated calcium currents were described in rat DHNs (Ryu and Randic, 1990), including one of the N-type (Huang, 1989). They appeared, however, insufficient to support plateau potentials, which were readily blocked in the presence of nifedipine. Another potential activator of $I_{\mathrm{CAN}}$ is the calcium released from the intracellular store (Zhang et al., 1995). In any case, it would have to be via a $\mathrm{Ca}^{2+}$-induced $\mathrm{Ca}^{2+}$ release (for review, see Simpson et al., 1995) consecutive to the activation of L-type VGCC. These additional possibilities require further studies.

Firing acceleration, maximum firing frequency, and duration of the afterdischarge are rather variable depending on the neuron (Russo and Hounsgaard, 1996; Morisset and Nagy, 1998). This variability is such that it often prevents normalized quantification of the effects of channel blockers (Figs. 5, compare $A, B ; 6$, compare $A, B)$. This indicates that, in the balance of the intrinsic conductances, the relative weight of L-type $I_{\mathrm{Ca}}$ and $I_{\mathrm{CAN}}$, and as stressed for turtle DHNs (Russo and Hounsgaard, 1996) of the $\mathrm{Ca}^{2+}$-dependent $\mathrm{K}^{+}$current, is probably set differently in different neurons.

In summary, in DHNs of the rat spinal cord, an L-type calcium current is the principal depolarizing component during the early phase of the plateau potential, ensuring the initial regenerative depolarization and firing acceleration. It subsequently triggers and maintains a CAN current, responsible for the high-frequency firing, and expression of prolonged afterdischarge. Regenerative properties of the rat DHNs, therefore, appear more complex than those described for the same class of spinal neurons and for motoneurons in the turtle in which the plateau potential is supported essentially by a noninactivating L-type current (Hounsgaard and Mintz, 1988; Russo and Hounsgaard, 1996).

\section{Plateau potential-mediated $\mathrm{Ca}^{2+}$ influx and pain processing in spinal cord}

As reported previously, plateau-generating cells in the deep dorsal horn are comprised preferentially of wide-dynamic range neurons and, to a smaller extent, of nociceptive specific neurons (Morisset and Nagy, 1998). The regenerative depolarizations associated with plateau potentials of these neurons are function- ally important for nociceptive integration in the spinal cord. They introduce nonlinearity in the information processing and enable the production of intense firing and afterdischarges in response to stimulation of nociceptive inputs (Morisset and Nagy, 1996, 1998; Russo and Hounsgaard, 1996). We have shown in the present paper that $\mathrm{Ca}^{2+}$ acts as a charge carrier participating in these prolonged depolarizations. However, intracellular calcium may act also as a second messenger directly and by stimulation of other second messenger systems. These effects, including synaptic plasticity and regulation of neuronal gene expression, are potentially of importance in pain processing (for review, see Woolf, 1996). $\mathrm{Ca}^{2+}$ influx during plateau potentials must contribute substantially to elevate the internal calcium concentration.

Additional support for the functional importance of $\mathrm{Ca}^{2+}$ dependent plateau potentials of DHNs in processing of nociceptive information comes from in vivo studies showing that L-type VGCCs are involved in the hyperalgesia and allodynia resulting from various nociceptive stimulations and inflammatory conditions in the rat (Martin et al., 1996; Neugebauer et al., 1996; Sluka, 1997). Interestingly, L-type calcium channels might not mediate electrically evoked synaptic release of transmitters (Holz et al., 1988; Takahashi and Momiyama, 1993), leaving for the plateau-mediated calcium influx a potential role in the $\mathrm{Ca}^{2+}$ mediated DHNs sensitization.

\section{REFERENCES}

Angstadt JD, Choo JJ (1996) Sodium-dependent plateau potentials in cultured retzius cells of the medicinal leech. J Neurophysiol 76:1491-1502.

Asada H, Yamaguchi Y, Tsunoda S, Fukuda Y (1996) Relation of abnormal burst activity of spinal neurons to the recurrence of autotomy in rats. Neurosci Lett 213:99-102.

Avery RB, Johnston D (1996) Multiple channel types contribute to the low-voltage-activated calcium current in hippocampal CA3 pyramidal neurons. J Neurosci 16:5567-5582.

Bal T, McCormick DA (1993) Mechanisms of oscillatory activity in guinea-pig nucleus reticularis thalami in vitro: a mammalian pacemaker. J Physiol (Lond) 468:669-691.

Beurrier C, Congar P, Bioulac B, Hammond C (1999) Subthalamic nucleus neurons switch from single-spike activity to burst- firing mode. J Neurosci 19:599-609.

De Koninck Y, Henry JL (1991) Substance P-mediated slow excitatory 
postsynaptic potential elicited in dorsal horn neurons in vivo by noxious stimulation. Proc Natl Acad Sci USA 88:11344-11348.

Fox AP, Nowycky MC, Tsien RW (1987a) Kinetic and pharmacological properties distinguishing three types of calcium currents in chick sensory neurones. J Physiol (Lond) 394:149-172.

Fox AP, Nowycky MC, Tsien RW (1987b) Single-channel recordings of three types of calcium channels in chick sensory neurones. J Physiol (Lond) 394:173-200.

Fraser DD, MacVicar BA (1996) Cholinergic-dependent plateau potential in hippocampal CA1 pyramidal neurons. J Neurosci 16:4113-4128.

Gerber G, Cerne R, Randic M (1991) Participation of excitatory amino acid receptors in the slow excitatory synaptic transmission in rat spinal dorsal horn. Brain Res 561:236-251.

Golowasch J, Marder E (1992) Ionic currents of the lateral pyloric neuron of the stomatogastric ganglion of the crab. J Neurophysiol 67:318-331.

Grubb BD, Riley RC, Hope PJ, Pubols L, Duggan AW (1996) The burst-like firing of spinal neurons in rats with peripheral inflammation is reduced by an antagonist of $N$-methyl-D-aspartate. Neuroscience 74:1077-1086.

Holz GG, Dunlap K, Kream RM (1988) Characterization of the electrically evoked release of substance $\mathrm{P}$ from dorsal root ganglion neurons: methods and dihydropyridine sensitivity. J Neurosci 8:463-471.

Hounsgaard J, Mintz I (1988) Calcium conductance and firing properties of spinal motoneurones in the turtle. J Physiol (Lond) 398:591-603.

Hsiao C-F, Del Negro CA, Trueblood PR, Chandler SH (1998) Ionic basis for serotonin-induced bistable membrane properties in guinea pig trigeminal motoneurons. J Neurophysiol 79:2847-2856.

Huang L-YM (1989) Calcium channels in isolated rat dorsal horn neurones, including labelled spinothalamic and trigeminothalamic cells. J Physiol (Lond) 411:161-177.

Kavalali ET, Plummer MR (1996) Multiple voltage-dependent mechanisms potentiate calcium channel activity in hippocampal neurons. J Neurosci 16:1072-1082.

Kiehn O (1991) Plateau potentials and active integration in the "final common pathway" for motor behaviour. Trends Neurosci 14:68-73.

Kiehn O, Harris-Warrick RM (1992) Serotonergic stretch receptors induce plateau properties in a crustacean motor neuron by a dualconductance mechanism. J Neurophysiol 68:485-495.

Klink R, Alonso A (1997) Ionic mechanisms of muscarinic depolarization in entorhinal cortex layer II neurons. J Neurophysiol 77:1829-1843.

Laird JMA, Bennett GJ (1993) An electrophysiological study of dorsal horn neurons in the spinal cord of rats with an experimental peripheral neuropathy. J Neurophysiol 69:2072-2085.

Marchetti C, Amico C, Usai C (1995) Functional characterization of the effect of nimodipine on the calcium current in rat cerebellar granule cells. J Neurophysiol 73:1169-1180.

Martin MI, Del Val VL, Colado MI, Goicoechea C, Alfaro MJ (1996) Behavioral and analgesic effects induced by administration of nifedipine and nimodipine. Pharmacol Biochem Behav 55:93-98.

Mills JD, Pitman RM (1997) Electrical properties of a cockroach motor neuron soma depend on different characteristics of individual $\mathrm{Ca}$ components. J Neurophysiol 78:2455-2466.

Morisset V, Nagy F (1996) Modulation of regenerative membrane properties by stimulation of metabotropic glutamate receptors in rat deep dorsal horn neurons. J Neurophysiol 76:2794-2798.

Morisset V, Nagy F (1998) Nociceptive integration in the rat spinal cord: role of nonlinear membrane properties of deep dorsal horn neurons. Eur J Neurosci 10:3642-3652.

Murase K, Ryu PD, Randic M (1989) Tachykinins modulate multiple ionic conductances in voltage-clamped rat spinal dorsal horn neurons. J Neurophysiol 61:854-865.

Nagy I, Maggi CA, Dray A, Woolf CJ, Urban L (1993) The role of neurokinin and $N$-methyl-D-aspartate receptors in synaptic transmission from capsaicin-sensitive primary afferents in the rat spinal cord in vitro. Neuroscience 52:1029-1037.

Neugebauer V, Vanegas H, Nebe J, Rümenapp P, Schaible HG (1996) Effects of $\mathrm{N}$ - and $\mathrm{L}$-type calcium channel antagonists on the responses of nociceptive spinal cord neurons to mechanical stimulation of the normal and the inflamed knee joint. J Neurophysiol 76:3740-3749.
Overton PG, Clark D (1997) Burst firing in midbrain dopaminergic neurons. Brain Res Rev 25:312-334.

Palecek J, Paleckova V, Dougherty PM, Carlton SM, Willis WD (1992) Responses of spinothalamic tract cells to mechanical and thermal stimulation of skin in rats with an experimental peripheral neuropathy. J Neurophysiol 67:1562-1573.

Partridge LD, Muller TH, Swandulla D (1994) Calcium-activated nonselective channels in the nervous system. Brain Res Rev 19:319-325.

Rekling JC, Feldman JL (1997) Calcium-dependent plateau potentials in rostral ambiguous neurons in the newborn mouse brain stem in vitro. J Neurophysiol 78:2483-2492.

Russo RE, Hounsgaard J (1996) Plateau-generating neurones in the dorsal horn in an in vitro preparation of the turtle spinal cord. J Physiol (Lond) 493:39-54.

Russo RE, Nagy F, Hounsgaard J (1997) Modulation of plateau properties in dorsal horn neurones in a slice preparation of the turtle spinal cord. J Physiol (Lond) 499:459-474.

Ryu PD, Randic M (1990) Low- and high-voltage-activated calcium currents in rat spinal dorsal horn neurons. J Neurophysiol 63:273-285.

Sandler VM, Puil E, Schwarz DWF (1998) Intrinsic response properties of bursting neurons in the nucleus principalis trigemini of the gerbil. Neuroscience 83:891-904.

Shaw T, Lee RJ, Partridge LD (1995) Action of diphenylamine carboxylate derivatives, a family of non-steroidal anti-inflammatory drugs, on $\left[\mathrm{Ca}^{2+}\right]_{\mathrm{i}}$ and $\mathrm{Ca}^{2+}$-activated channels in neurons. Neurosci Lett 190:121-124.

Simpson PB, Challiss RAJ, Nahorski SR (1995) Neuronal $\mathrm{Ca}^{2+}$ stores: activation and function. Trends Neurosci 18:299-306.

Sluka KA (1997) Blockade of calcium channels can prevent the onset of secondary hyperalgesia and allodynia induced by intradermal injection of capsaicin in rats. Pain 71:157-164.

Sotgiu ML, Biella G, Riva L (1995) Poststimulus afterdischarges of spinal WDR and NS units in rats with chronic nerve constriction. NeuroReport 6:1021-1024.

Swandulla D, Lux HD (1985) Activation of a nonspecific cation conductance by intracellular $\mathrm{Ca}^{2+}$ elevation in bursting pacemaker neurons of Helix pomatia. J Neurophysiol 54:1430-1443.

Takahashi T, Momiyama A (1993) Different types of calcium channels mediate central synaptic transmission. Nature 366:156-158.

Tsien RW, Lipscombe D, Madison DV, Bley KR, Fox AP (1988) Multiple types of neuronal calcium channels and their selective modulation. Trends Neurosci 11:431-438.

Urban L, Randic M (1984) Slow excitatory transmission in rat dorsal horn: possible mediation by peptides. Brain Res 290:336-341.

Viana di Prisco G, Pearlstein E, Robitaille R, Dubuc R (1997) Role of sensory-evoked NMDA plateau potentials in the initiation of locomotion. Science 278:1122-1125.

Wilson GF, Richardson FC, Fisher TE, Olivera BM, Kaczmarek LK (1996) Identification and characterization of $\mathrm{a} \mathrm{Ca}^{2+}$-sensitive nonspecific cation channel underlying prolonged repetitive firing in Aplysia neurons. J Neurosci 16:3661-3671.

Woolf CJ (1996) Windup and central sensitization are not equivalent. Pain 66:105-108.

Woolf CJ, King AE (1987) Physiology and morphology of multireceptive neurons with $\mathrm{C}$-afferent fiber inputs in the deep dorsal horn of the rat lumbar spinal cord. J Neurophysiol 58:460-479.

Yoshimura M, Jessell TM (1990) Amino acid-mediated EPSPs at primary afferent synapses with substantia gelatinosa neurones in the rat spinal cord. J Physiol (Lond) 430:315-335.

Yoshimura M, Shimizu T, Yajiri Y, Inokuchi H, Nishi S (1993) Primary afferent-evoked slow EPSPs and responses to substance P of dorsal horn neurons in the adult rat spinal cord slices. Regul Pept 46:407-409.

Zhang B, Harris-Warrick RM (1995) Calcium-dependent plateau potentials in a crab stomatogastric ganglion motor neuron. I. Calcium current and its modulation by serotonin. J Neurophysiol 74:1929-1937.

Zhang B, Wootton JF, Harris-Warrick RM (1995) Calcium-dependent plateau potentials in a crab stomatogastric ganglion motor neuron. II. Calcium-activated slow inward current. J Neurophysiol 74:1938-1946. 